\title{
An access control framework for web services
}

\author{
M. Coetzee \\ J.H.P. Eloff
}

M. Coetzee, School of Information Technology, University of Johannesburg, Johannesburg, South Africa

J.H.P. Eloff, Information and Computer Security Architectures (ICSA) Research Group, Department of Computer Science, University of Pretoria, Pretoria, South Africa

The financial assistance of the Department of Labour (DoL) towards this research is hereby acknowledged. Opinions expressed and conclusions arrived at, are those of the author and are not necessarily to be attributed to the DoL.

[The figures and tables at the bottom of this document]

Purpose - To define a framework for access control for virtual applications, enabled through web services technologies. The framework supports the loosely coupled manner in which web services are shared between partners.

Design/methodology/approach - A background discussion on relevant literature, with an example is used to illustrate the problem that exists. To enable access control composition, an extension is proposed to authorisation specification language, together with publication of access control requirements of a web service provider.

Findings - The framework shows that loosely coupled access control can be made possible by making use of the standard manner in which messages are communicated in $\mathrm{XML}$, and by composing assertions with the access control policy of the provider in a consistent manner. Access to web service methods is only granted if permission can be derived for it, where the derivation step forms a formal proof.

Research limitations/implications - A basic framework has been defined. An architecture to support it must be defined. Only a very basic level of access control composition has been illustrated.

Practical implications - The publication of access control requirements in standards such as WS-Policy can be considered.

Originality/value - This paper offers a practical approach to address access control for web services. 


\section{Introduction}

Advances in the internet have transformed distributed systems into a marketplace of services that can be shared across organisational boundaries. A recent trend in information technology is business-to-business collaboration, where business functionality is supported through virtual applications. Business functionality of organizations is becoming more digitised, and software characteristics such as virtuality and real-time operation is exhibited. Virtual applications are enabled by web services technology (Gottschalk et al., 2002) that allows organizations to exploit software as a service, through service virtualization. Web services is also recognized as the logical architecture for the organization of grid services (Myer, 2003). Security would be an important consideration in the design of such networked services.

Web services are based on a set of XML standards such as simple object access protocol (SOAP) (Box et al., 2000), web service definition language (WSDL) (Christensen et al., 2001), and universal description, discover and integration (UDDI) (Atkinson et al., 2003). A web service is the name of an object with methods that can be invoked through an internet connection. Web services providers define XML-based interfaces with WSDL. This enables RPC mechanisms, based on network endpoints, to exchange SOAP messages with web services requestors. The dynamic assembly of software and resources, written in different programming languages and tools, residing on different operating systems can then occur. Assembly is between applications on a machine-tomachine basis and appears virtual to the human utilising such functionality. Loose coupling is enabled as web services providers publish well-defined, extensible interfaces, and web services requestors locate them and submit requests as platform independent, self-describing, self-contained messages, shown in Figure 1.

The use of web services technology presents new security problems to IT departments of organizations. A unique characteristic of web services is that they allow business partners to communicate through human-legible SOAP messages. A partner's secrets may be exposed in a SOAP response and must be protected from threats such as disclosure to unauthorized parties. Owing to the fact that XML is text-based, web services invocations can pass over normal HTTP channels and therefore through firewalls. The applications that are to process the request contained by these XML messages may then be endangered by false claims or malicious information.

The distributed, loosely coupled architecture of virtual applications present challenges in terms of verifying credentials and exercising access control over diversified resources. Even though access control for web services has been researched (Damiani et al., 2001), there is not yet a defined architecture for access control when web services are composed into a virtual application. The focus of this paper is to define a framework for access control integration for networked web services. Such a framework would need to support the loosely coupled manner in which these services are shared between business partners. This paper is structured as follows. Section 2 provides a background to the problem with an example. Section 3 addresses the access control of a virtual application. Section 4 concludes the paper. 


\section{Background}

XML has become a standard for communication between applications. With XML, an application defines markup tags to represent the different elements of data in a text file so that the data can be read and processed by any application that uses XML.

SOAP allows the structuring of data in XML so that different applications can read and send messages, generally over HTTP, to each other. SOAP messages contain three different sections, as shown in Figure 2. Their semantics are defined with associated XML schemas, which the receiving SOAP processor should be able to interpret. The SOAP Envelope element defines the start and end of the message. The SOAP Header element, which is optional, allows data not directly associated with a method request or response to be passed to a web service. The SOAP Body element contains the web services method name and associated parameters in either the SOAP request or response. To secure exchanges, message security can be added to the SOAP message by extending the SOAP header with security information.

Conceptually, information security is enforced by means of information security services such as authentication, authorization, confidentiality and integrity. A number of new specifications have been developed to embed these security services within the SOAP header, to ensure the security of a message between intermediate and destination security domains. Security assertion markup language (SAML) (Hallam-Baker et al., 2002) is a token-passing system to securely exchange authentication and authorization information. XML Signature (Bartel et al., 2002) and XML Encryption (Imamura et al., 2002) describe how integrity and confidentiality are enforced for XML documents, while WSSecurity (Atkinson et al., 2002) applies such XML security technologies to SOAP messages with XML elements describing credential exchange, message integrity and message confidentiality. XML access control markup language (XACML) (Anderson et al., 2003) allows fine-grained access control policies to be expressed in XML. To secure SOAP messages, a combination of XML Signature, XML Encryption, SAML, and XACML can be used to ensure authentication, authorization, integrity and confidentiality.

A web service belongs to a specific organization, with its own distinctive access control policies and models. To allow interoperation between independent security domains, WSPolicy (Box et al., 2003) provides a grammar for requestors and providers of web services to communicate their requirements and capabilities. In addition, WS-Trust (Anderson et al., 2004) describes a framework for managing, assessing and establishing trust relationships to enable web services to interoperate securely.

Specifications that address authentication and access control are not established to the extent as those for the integrity and confidentiality of SOAP messages. As application interfaces are exposed, web services access control is important to implement. Access control should not only be required for information, but the operations performed by remote users or applications should be carefully controlled. Because remote users or 
applications will have access to an increasing number of services without human checkpoints, access control should be actively managed.

\section{eCompany sales - an example}

Web services allow virtual application partners to interact with others in an ad hoc fashion, at distant, independent locations. Consider as an example eCompany sales, a virtual application shown in Figure 3. eRetailer is a provider of a retail web service. eCompany, a web service requestor, enables its users to place orders for goods at eRetailer. eCompany can provide more sophisticated services to their customers and employees, by integrating the services of eRetailer with its own business functionality. A benefit to eCompany is that it may receive a percentage of the profit made on the sales done by its customers and employees.

eCompany sales can only be successful if it allows customers and employees to navigate easily and securely between the web sites supporting these services. There also needs to be some relationship of trust between eCompany and eRetailer, as this will form the basis of all exchanges that may take place between them. This can be defined when eCompany registers with eRetailer through a register_business service. A designated employee does this on behalf of the eCompany requestor application. In the open environment in which eCompany sales exists, an identifying digital certificate may be required, or an identifying token issued, that will be used when requests are processed. Thereafter, services are made available to the employees and customers of eCompany such as searching through products and special offers on to-be-released products, adding selected products to a shopping basket and placing of orders. To the customers and employees of eCompany, the eCompany sales virtual application would seem local to their organisation. To be effective, it would require access control to be enforced across domains.

\section{Access control for eCompany sales}

A virtual application is an environment where both the requestor and provider of the service may require the ability to influence the access control decisions that are made when the provider processes a request. Composing access control policies between the requestor and provider is challenging, as there is no support available to administrators to address this problem.

To describe the problem of access control composition, consider the following: eRetailer hosts a very generic retail service to accommodate any number of service requestors. eRetailer can provide more flexibility to service requestors, by giving them limited control over the type of interaction that would occur. For instance, special offers may be made available to customers on to-be-released products. This is a benefit that a service 
requestor may pass on to their customers when orders for selected products are processed, but which will negatively impact the percentage of profit made by the service requestor. Even though this is a choice made by the management of a service requestor, it has to be applied by the service at eRetailer. eCompany may decide to provide this benefit to their employees, but not to their customers. If Jill, an employee of eCompany is given this benefit, she should have permission to execute the remote list_specials method of the service at eRetailer. John, a customer of eCompany will not be given this benefit. John would only be able to execute the product_search method. John and Jill are not known to eRetailer. eRetailer would only be able to differentiate between their requests, if additional information is provided by eCompany. As eCompany and eRetailer each have their own authentication mechanisms, costly investments could be avoided by allowing eCompany to do its own authentication, but to provide loose coupling of authentication with assertions, defined in XML. eRetailer may also not be interested in the real identity of John or Jill, but rather in their ability or role requested on their behalf by eCompany, as the request is processed. Such an assertion is a claim, statement or declaration of fact made by eCompany, and is not necessarily signed (Bonatti and Samarati, 2002).

Access control information such as credentials (Samarati, 2002) and assertions that are passed to the eRetailer service provider, must be composed with its access control decision-making process. This can be achieved if policies are defined in a declarative manner that is machine-readable. Logical languages are attractive as policy specification languages, as a good compromise between expressiveness and simplicity can be achieved (Bonatti and Samarati, 2003). Access control permissions need to be defined, with access control logic that would be able to infer consistent decisions over objects, to which more than one policy applies. It could be argued that all such decisions should rather be left to the requestor, to be decided before a request is sent, and that interactions should be made as simple as possible, as to ensure a very generic service that could be used by various types of client applications. In simple cases this may be true. But, when the components of a virtual application are coupled, it may become necessary to enable flexible decisions through the composition of access control information and policies from different sources.

When considering the composition of access control policies across organisational boundaries, role-based access control (RBAC) can be useful as it reduces the administration burden (Sandu, 1996). RBAC requires access permissions to be assigned to roles, rather than individual users, and users obtain permissions by virtue of being assigned appropriate roles. Recently, the composition of independent role-based access control policies between organisations has been addressed (Lischka and Wedde, 2003). Such composition leads to tight intersystem coupling, as there should be agreements between web services requestors and providers, to define which role can use which web service and to what extent. Loose coupling and late binding are concepts central to web services. If access control is implemented by implementing access control policies at both the provider and requestor, and these policies require substantial development effort for each new change made to the policies, it would become the antithesis of loose coupling. Access control information should also not be made part of the interface of a particular web service, but should be defined in a standard manner, that would ensure re-use of 
access control semantics across participants of a virtual application. Here, loosely coupled access control is enabled, by publishing the web services provider roles that can be activated by a requestor. In addition, role membership or privileges can dynamically be adapted by the requestor, as is made possible by the provider. The next two paragraphs will discuss how this can be implemented.

\section{Loosely coupled access control through publicly defined roles}

WSDL is a mechanism that supports loose coupling and late binding for web services. But, as WSDL does not support security, it does not provide a complete solution. A web service provider belongs to a specific organization, with its own distinctive access control policies and models. Its security expectations should also be made available to allow interoperation with web services requestors. WS-Policy (Box et al., 2003) provides a grammar for requestors and providers of web services to communicate their requirements and capabilities in a machine-readable XML format. A policy is defined to be a collection of one or more policy assertions. The XML representation of a policy is referred to as a policy expression, and is bound to a web service provider through a policy attachment. WS-Policy defines the security requirements related to authentication, integrity and confidentiality of SOAP messages, and can be attached to the WSDL service interface to describe security expectations. No expectations in terms of access control are currently described.

A new extension to WS-Policy can be included that would provide all or some of the access control policy requirements to potential requestors so that they can make decisions regarding if and how they can use methods of the service. An optional

AccessControlPolicy element can include some authorization information of the provider that can be used to facilitate access control composition. For instance, eRetailer may publish a list of roles, with their descriptions such as Standard_Customer and Gold_Customer as shown in Figure 4. Such published roles may provide generic functionality with associated privileges that are described in the RoleDescription element in Figure 4. To enable this, a request from eCompany must be accompanied with an assertion stating the role that must be activated for the employee or customer. It may consist out of two name-value pairs such as: Attribute name="Role”, Attribute value="Standard_Customer" that can be standardised by a provider defined schema at www.eRetailer.com.

As role information is published, no formal interactions or agreements are required with eRetailer. eCompany is thus given more control over the access control decisions that will be made when the services of eRetailer are processed. Other access control information that can be published is, for instance, attributes such as the limit that may be spent by the user. As the manner in which policy assertions are to be formulated is published, the web service requestor can define assertions that would be understood by the provider. Such assertions need to be pushed to the web service provider, to simplify the processing of access control decisions and to avoid unnecessary communication with 
the requestor. This would require a mechanism that would allow such access control information to pass between services. Security assertion markup language (SAML) may be well suited for this purpose, as it can be used to securely exchange authentication and authorization information.

\section{Dynamic composition of access control information from independent authorities}

A loosely coupled, distributed environment enables dynamic access control composition by sending assertions between participants of a virtual application. A transaction can only be processed if the defined access control decision-making process can compose the web service provider's policy with the requestor's assertions. Currently, the composition of such access control communication is beyond the scope of existing access control mechanisms. It would therefore, be entirely up to the web services developer to enforce access control policies with the appropriate logic in application code. This could result in error-prone implementation, as an omission or misinterpretation of a communication from another security domain may lead to improper access to resources.

In contrast, a logic-based access control system has the formal foundation of logical reasoning, to enable the enforcement of consistent access control decisions over resources of the virtual application. A virtual access control policy can be created, that has the ability to dynamically adapt to changes passed to it from independent policy sources. It is an access control policy that can be understood and applied by the authorization manager of a virtual application. The authorization semantics of the policy are defined independently of the policy representation and implementation mechanisms. It consists of a set of facts such as the permission assigned to roles, and rules on how roles may be activated. In logic programming, an assertion is defined as a new fact or rule that is added to the program at run time (Foldoc, 2003). Assertions that are sent across from other domains can therefore be logically composed with the existing virtual policy as additional facts or rules. In this process, new assertions are formally proven to be valid, before access control decisions are made, that are based on these assertions.

\section{Logical composition of assertions}

There have been a number of attempts to declaratively define access control policies in first-order logic. Most of these approaches are based on some variant of Datalog (Elsmari and Navathe, 2000). Authorization specification language (ASL) (Jajodia et al., 2001) is a formal logic language for specifying access control policies. ASL supports a variety of access control models, but was not originally designed to enable dynamic policy composition. Policies are expressed by forming rules that use the described predicates. For instance, cando(o, s, sa) defines the signed authorization (sa) explicitly inserted by the security officer for a subject (s) on an object (o); dercando(o, s, sa) defines 
signed authorization derived by the system using logical rules of inference; and active(u, r) defines the role active for a subject.

In a web services environment, the provider cannot mandate any particular access control models or mechanisms at the requestor. It would also be impractical to encode all access control rules from each domain into a centralized ASL program that would make centralised decisions for the virtual application. Here, a policy is defined in ASL for the web service provider. The specific subjects, objects and actions to be defined are:

- Objects. The WSDL service description of a web service contains all methods that can be executed. Methods belong to objects, which can be grouped together to form virtual applications. Other objects that could be considered would be the server that the web service resides on, the IP address, or the URL of the web service.

- Subjects. A distinction exists between the subjects with specified permissions and the active subjects requiring access to objects. For instance, a user of a virtual application is an authorization subject whereas a requestor method, acting on behalf of users is an active subject (Bertino et al., 2000). Here an active subject is referred to as a requestor.

- Actions. Remote users or applications would generally be allowed to execute a web services method, or access a server hosting a number of web services objects. A requirement of such an environment would be to dynamically revoke permissions when needed.

A simple list of role-based access control permissions for the product_search and list_specials methods of the eRetailer service may exist as follows: Equation 1, Equation 2 and Equation 3 The first and second rule assigns the permission to execute the product_search method to the Standard_Customer and Gold_Customer roles. The third rule assigns the permission to execute the list_specials method to the Gold_Customer role.

ASL is extended with predicates to enable dynamic composition. An assertion can only be imported if there exists a relationship of trust between the web service requestor and provider. This is a fact that is added as trust (Requestor), as shown by statement 4 below. An identifying token or public key can identify the web service requestor. Such a fact is added when the business registration process is done. If the provider loses trust in the requestor, the statement can immediately be removed from the set of facts, and no assertions sent by the requestor would successfully be derived as new facts. Equation 4 If Jill, an employee of eCompany wishes to list all specials, eCompany sends an assertion to eRetailer, requesting that the list_specials method be executed as a Gold_Customer. eRetailer imports the assertion by converting the XML assertion into a logical statement with the request predicate, to distinguish it from local facts. $\mathrm{R}$ request $\mathrm{f}$ means that the requestor $r$ requests formula $f$ to be added as a new fact or rule. Each request made to the provider in an assertion should also include the identifying token or certificate. The XML assertion is programmatically converted into a logical fact as: Equation 5 Roles active for a requestor are derived by: Equation 6 Permissions that include imported facts can be 
derived by: Equation 7 Jill is granted permission to execute the list_special method, as eCompany is trusted by eRetailer, and permission can be derived for the activated role.

An important consideration would be the management of the lifespan of an imported fact. Imported facts can be evaluated based on the time associated with it, and can be removed when expired.

\title{
Conclusion
}

Here, an access control framework was defined, to address the loosely coupled manner in which virtual applications are created. As such applications may exist for very limited time periods, or have a limited number of transactions, they should be composed quickly, with embedded flexibility. The framework makes use of the standard manner in which messages are communicated in XML between disparate applications, but composes such assertions with the access control policy of the provider in a consistent manner. Access to web service methods is only granted if permission can be derived for it, where the derivation step forms a formal proof. A very simple example of composition was illustrated. Future research would include the composition of the provider access control policy, with authorization assertions from other domains, and default rules that may apply to the virtual application.

\author{
Equation 1 \\ cando(product_search, Standard_Customer, +execute) \\ Equation 2 \\ cando(product_search, Gold_Customer, +execute) \\ Equation 3 \\ cando(list_specials, Gold_Customer, +execute)
}

Equation 4

trust(XC55674XX) 


\section{Equation 5}

requestor(XC55674XX)request activate(Gold_Customer)

\section{Equation 6}

active(Requestor, Role)

$\leftarrow$ trust(Requestor) \& requestor(Requestor)requests activate(Role)

\section{Equation 7}

dercando(Object, Role, SAction)

$\leftarrow$ active(Requestor, Role) \& cando(Object, Role, SAction)

Figure 1 Tightly coupled vs loosely couple integration

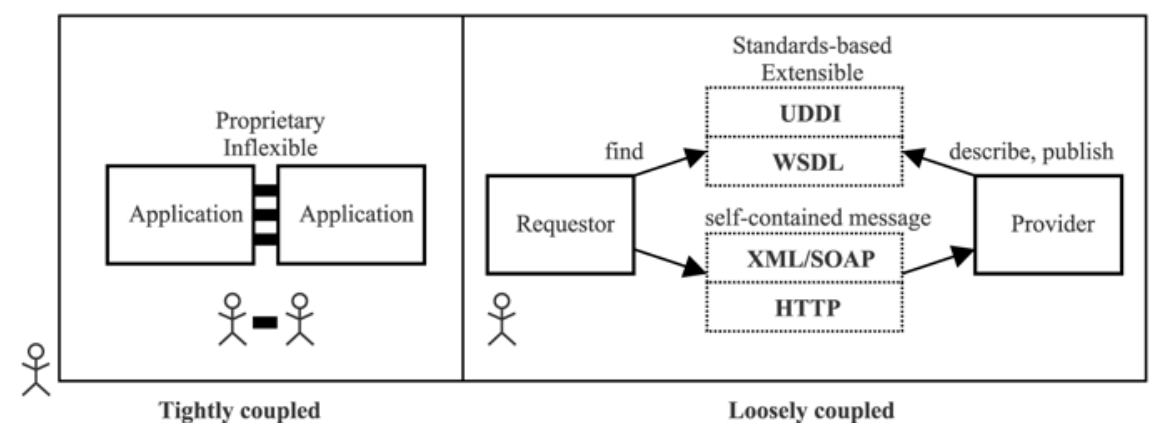

Figure 2 A SOAP message with authentication information defined with WS-security

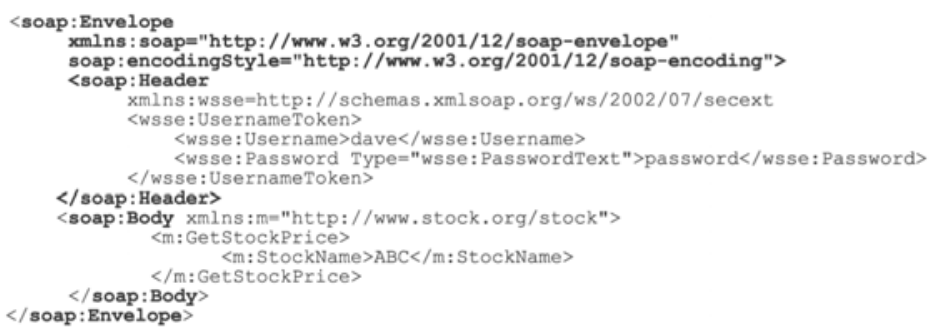


Figure 3 A virtual application

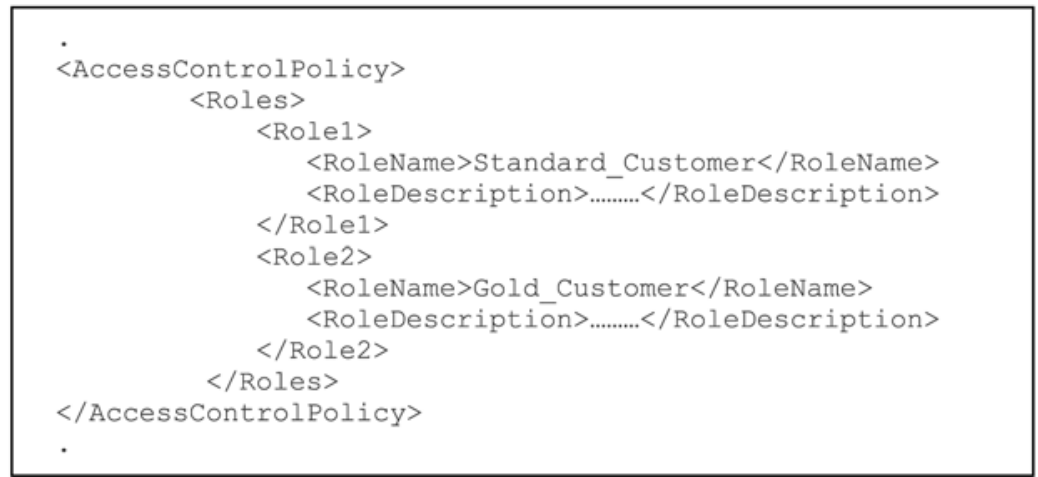

Figure 4 An extension of a WS-policy requirements description in XML

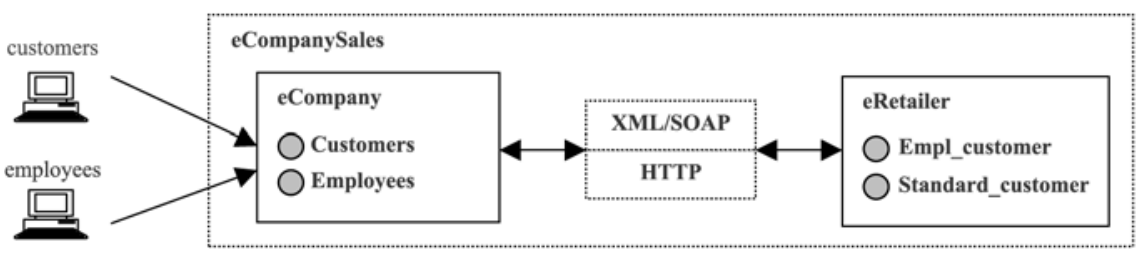

\section{References}

Anderson, A., Anderson, S., Adams, C., Beznosov, K., Brose, G., Crocker, S. (2003), Extensible Access Control Markup Language (XACML) 1.0 Specification, available at: www.oasis-open.org/committees/tc_home.php?wg_abbrev=xacml, .

Anderson, S., Bohren, J., Boubez, T., Chanliau, M., Della-Libera, G., Dixon, B. (2004), Web Services Trust Language (WS-Trust), available at: www.ibm.com/developerworks/library/ws-trust/index.html, .

Atkinson, A., Bellwood, T., Cahuzac, M., Clément, L., Colgrave, J., Corda, U. (2003), UDDI Version 3.0.1, available at: http://uddi.org/pubs/uddi-v3.0.1-20031014.htm, .

Atkinson, B., Della-Libera, G., Hada, S., Hondo, M., Hallam-Baker, P., Kaler, C. (2002), Web Services Security (WS-Security) Version 1.0, available at: www.verisign.com/wss/wss.pdf (accessed 5 April), . 
Bartel, M., Boyer, J., Eastlake, D., Fox, B., LaMacchia, B., Simon, E., Solo, D. (2002), XML Signature, available at: www.w3.org/TR/2002/REC-xmldsig-core-20020212/, .

Bertino, E., Pagani, E., Rossi, G.P., Samarati, P. (2000), "Protecting information on the web", Communications of the ACM, Vol. 43 No.11, pp.189-99.

Bonatti, P., Samarati, P. (2002), "A unified framework for regulating access and information release on the web", Journal of Computer Security, Vol. 10 No.3, pp.241-72.

Bonatti, P., Samarati, P. (2003), "Logics for authorizations and security", in Chomicki, J., van der Meyden, R., Saake, G. (Eds),Logics For Emerging Applications of Databases LNCS, Springer-Verlag, Heidelberg, .

Box, D., Curbera, F., Hondo, M., Kale, C., Langworthy, D., Nadalin, A. (2003), Web Services Policy Framework (WS-Policy), available at: www.ibm.com/developerworks/library/ws-policy/index.html, .

Box, D., Ehnebuske, D., Kakivaya, G., Layman, A., Mendelsohn, N., Nielsen, H.F., Thatte, S., Winer, D. (2000), Simple Object Access Protocol (SOAP) 1.1, available at: www.w3.org/TR/SOAP/, .

Christensen, E., Curbera, F., Meredith, G., Weerawarana, S. (2001), Web Services Description Language (WSDL) 1.1, available at: www.w3.org/TR/wsdl, .

Damiani, E., De Capitani Di Vimercati, S., Paraboschi, S., Samarati, P. (2001), "Finegrained access control for SOAP e-services", Proceedings of the 10th International World Wide Web Conference, Hongkong, 1-5 May, .

Elsmari, R.A., Navathe, S. (2000), Fundamentals of Database Systems, Addison-Wesley, Milano, .

Foldoc (2003), Free, Online Dictionary of Computing, Supported by the Department of Computing Imperial College, available at: http://0-

foldoc.doc.ic.ac.uk.innopac.up.ac.za:80/foldoc/foldoc.cgi?query=assertion\&action=Searc h, .

Gottschalk, K., Graham, S., Kreger, H., Snell, J. (2002), "Introduction to web services architecture", IBM Systems Journal, Vol. 41 No.2, .

Hallam-Baker, P., Hodges, J., Maler, E., McLaren, C., Irving, R. (2002), SAML 1.0 Specification, available at: www.oasisopen.org/committees/tc_home.php?wg_abbrev=security, .

Imamura, T., Dillaway, B., Eastlake, D., Reagle, J., Simon, E. (2002), XML Encryption, available at: www.w3.org/TR/xmlenc-core/, . 
Jajodia, S., Samarati, P., Sapino, M.L., Subrahmanian, V.S. (2001), "Flexible support for multiple access control policies", ACM Transactions on Database Systems, Vol. 26 No.2, pp.214-60.

Lischka, M., Wedde, H.F. (2003), "Composing heterogenous access policies between organizations", Proceedings of the IADIS International Conference e-Society, Lisbon, 36 June, .

Myer, T. (2003), Grid Computing: Conceptual Flyover for Developers, available at: www-106.ibm.com/developerworks/library/gr-fly.html, .

Samarati, P. (2002), "Enriching access control to support credential-based specifications", paper presented at the Workshop-Credential-Based Access Control in Open, Interoperable IT-Systems, Dortmund, 2 October, available at: http://ls6-www.cs.unidortmund.de/issi/cred_ws/, .

Sandu, R. (1996), "Access control: the neglected frontier", Proceedings of the 1st Australian Conference on Information Security and Privacy, Wollongong, 23-26 June, pp.23-36. 